\title{
UNIVERSITYOF
}

FORWARD

THINKING

WESTMINSTER用

WestminsterResearch

http://www.westminster.ac.uk/westminsterresearch

Engaging immersive video consumers: Challenges regarding 360-degree gamified video applications

Argyriou, L., Economou, D., Bouki, V. and Doumanis, I.

This is a copy of the author's accepted version of a paper subsequently published in the proceedings of the 2nd International Workshop on Virtual Environments and Advanced Interfaces (VEAI 2016), within the the 15th International Conference on Ubiquitous Computing and Communications (IUCC-2016) . Granada, Spain, 14 to 16 December 2016.

It is available online at:

https://dx.doi.org/10.1109/IUCC-CSS.2016.028

(C) 2016 IEEE . Personal use of this material is permitted. Permission from IEEE must be obtained for all other uses, in any current or future media, including reprinting/republishing this material for advertising or promotional purposes, creating new collective works, for resale or redistribution to servers or lists, or reuse of any copyrighted component of this work in other works.

The WestminsterResearch online digital archive at the University of Westminster aims to make the research output of the University available to a wider audience. Copyright and Moral Rights remain with the authors and/or copyright owners.

Whilst further distribution of specific materials from within this archive is forbidden, you may freely distribute the URL of WestminsterResearch: ((http://westminsterresearch.wmin.ac.uk/)).

In case of abuse or copyright appearing without permission e-mail repository@westminster.ac.uk 



\section{Engaging immersive video consumers: Challenges regarding 360-degree gamified video applications}

\author{
Lemonia Argyriou \\ Department of Computer Science, \\ Faculty of Science \& Technology, \\ University of Westminster, \\ London, UK \\ argyrioulemonia@gmail.com
}

\author{
Daphne Economou \\ Department of Computer Science, \\ Faculty of Science \& Technology, \\ University of Westminster, \\ London, UK \\ D.Economou@westminster.ac.uk
}

\author{
Vassiliki Bouki \\ Department of Computer Science, \\ Faculty of Science \& Technology, \\ University of Westminster, \\ London, UK \\ boukiv@westminster.ac.uk
}

\author{
Ioannis Doumanis \\ CTVC Ltd, \\ London, UK \\ ioannis@truetube.co.uk
}

\begin{abstract}
-360-degree videos is a new medium that has gained the attention of the research community imposing challenges for creating more interactive and engaging immersive experiences. The purpose of this study is to introduce a set of technical and design challenges for interactive, gamified 360-degree mixed reality applications that immerse and engage users. The development of gamified applications refers to the merely incorporation of game elements in the interaction design process to attract and engage the user through playful interaction with the virtual world. The study presents experiments with the incorporation of series of game elements such as time pressure challenges, badges and user levels, storytelling narrative and immediate visual feedback to the interaction design logic of a mixed reality mobile gaming application that runs in an environment composed of 360-degree video and 3D computer generated objects. In the present study, the architecture and overall process for creating such an application is being presented along with a list of design implications and constraints. The paper concludes with future directions and conclusions on improving the level of immersion and engagement of 360-degree video consumers.
\end{abstract}

Keywords-user-centred design; 360-degree video; interaction design; immersion; games; design challenges;

\section{INTRODUCTION}

The desire for better immersion and presence in computer simulated environments has driven an aggressive growth of immersive technologies, presenting new forms of Virtual Reality (VR) media and innovative VR devices. A promising technology for experiencing VR is 360-degree video. 360degree video offers enhanced realism that although it is possible to produce using computer generated virtual environments, it is resource intensive and expensive process. This enhanced realism offered by video resources when combined with game techniques has great potentials of leading to highly immersive, interactive and engaging forms of experiencing VR. 360-degree video applications are based on free viewpoint videos and resemble navigation in virtual worlds of $3 \mathrm{D}$ computer graphics by allowing viewers to interactively change their viewpoint in the scene [27] while in traditional video productions the viewpoint is chosen by the director. At the moment, the interaction supported in such applications is restricted mainly to:
- the change of viewpoint by the user enabling them to look around in the 360-degree captured video scene;

- the selection of hyperlinks integrated in the video in different times and areas to: either load another 360degree video replacing the current scene giving the feeling of transitioning to another virtual environment; or load multimedia content that can either replace the current scene or could be overplayed in it.

In this paper we present the concept, preliminary set of technical and design challenges and implications for creating engaging and immersive 360-degree gamified video applications that enhance users' interaction with the content. These challenges are defined after a review on technical and design factors that affect the user experience in 360-degree video and the results of previous related work. We then demonstrate how those factors are addressed using an experimental case study. We conclude with discussion and future directions.

\section{BACKGROUND AND RELATED WORK}

There are two kinds of pleasure that users of interactive applications enjoy; immersion and engagement [5]. Immersion refers to the technical means that are used in order to give the user the notion of presence which relates to the perception of feeling present in a non-physical world [26]. 360-degree videos have a huge potential of generating highly immersive video environments that surround the user and offer an increased sense of presence. In addition to the benefits of 360-degree video, there are also two main characteristics of games that facilitate immersion and can be grouped into two general categories:

- those that create a rich mental model of the game environment, and;

- those that create consistency between the several elements of the environment, such as the game objects, the virtual actors, the interface elements etc. [29].

To support the creation of a rich mental model through 360degree gamified experiences, a cognitively demanding environment that triggers the user's thinking and understanding of what's going on in the scene should be designed. This can be achieved by devising interesting 360-degree branching 
narratives that attract user's attention and make the game world believable. Branching narrative belongs to the category of non-linear gameplay where players are presented with challenges that can be addressed through a number of different sequences. In branching, interactive narratives the player can select to follow different subplots of the game story which can lead to its success or failure at addressing a challenge [25].

In addition, to offer immersive gaming experiences, the game elements used should be consistent to the virtual environment [17]. In more detail, most important is that there are no incongruous visual cues in the game world like for example heads up displays, tutorial messages, distractive notifications, advertisements etc. Moreover, the behavior of virtual characters and objects in the game world should be believable, meaning that they should behave like you'd expect them to in the real world. Consistency in the gaming experience can be also achieved through the design and integration of interaction models that do create an unbroken presentation of the game world. Game menus and informative texts should not make the game world disappear. The design of the game's user interface (UI) should be non-distractive avoiding messages with long text and colors that will distract the player from the gameplay.

Another factor that can be also enhanced through gamification techniques is user engagement. The concept of gamification was introduced several years ago [19] as the use of game design elements in non-gaming applications and services to enhance the level of user's engagement and motivation. In contrast to serious games design that refers to complete gaming applications for non-entertainment purposes and has been widely used for educational and professional training purposes, gamified applications are not actually a game, but refer to the merely incorporation of game elements. Certain game elements when integrated in right combination can enhance the level of player's engagement [22]. Narrative context, time pressure, leaderboards, badges, level-systems, competition under strict rules, achievements and rewards are some of the mostly used game elements that are proven to drive the design of playful experiences. According to Andrezej Marczewki's diagram [18] as depicted in figure 1, the main differences between the terms game design, gamification, serious games or simulations and a complete game are based on the design goals and intentions which are game thinking, incorporation of game elements, game play or design just for fun experiences.

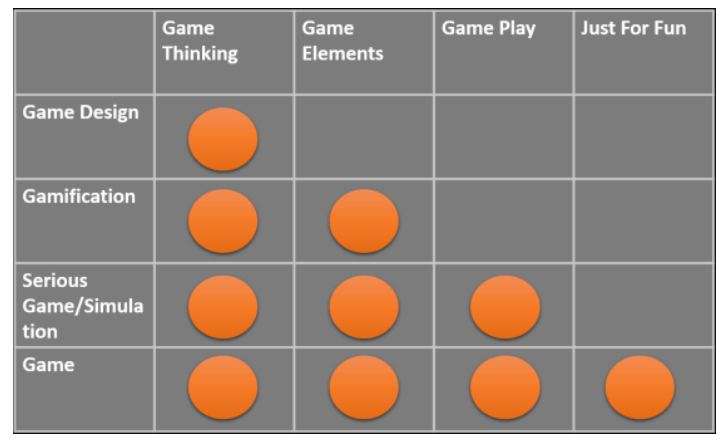

Fig. 1. Differences in Terms (game design, gamification, serious games, and games).
Another goal when designing gameful experiences, is targeting ludic engagement which, as defined by [9], is achieved through design and activities that are motivated by curiosity, exploration and reflection. Therefore, the overall design process of interactive applications towards providing enhanced immersive and engaging experiences can be based on the integration and right blending of 360-degree film-based narratives with interactive game elements.

A good example of the gamified 360-degree film-based narratives approach has been the award winning web applications of "Fort McMoney" [7] which is depicted in figure 2 below. In that case, game mechanics have been successfully integrated with traditional film production in order to engage citizens in actively taking part in the future development of their city (the construction of the world's largest sands reserves in the area of, Alberta, Canada and Athabasca).

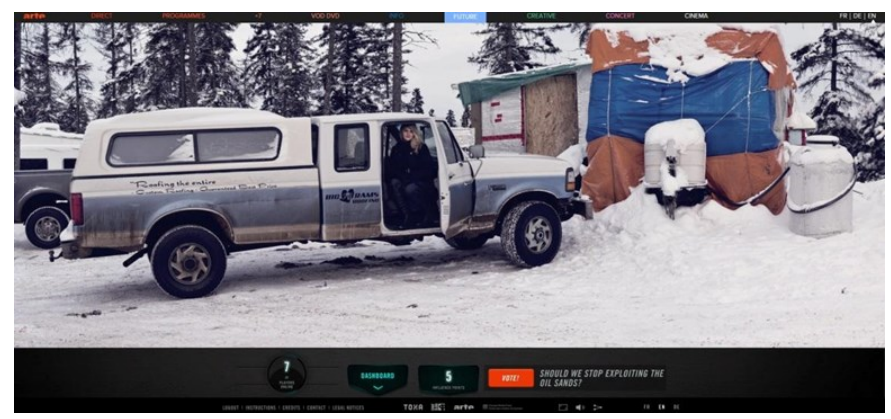

Fig. 2. Fortmcmoney an interactive Film-based serious game.

In Fort McMoney, the user can select to move in the story through hotspots that are presented at the end of a 360-degree video resource on a static panoramic image or through the user interface buttons at the bottom of its screen. Therefore through the complex interface and the use of static images that pause the film, the user can be distracted from the actual plot of the presented story and the sense of flow in the game can be lost. Moreover, this application has been designed for the web only therefore it cannot be experienced though a VR headset device such as Oculus Rift [20] which enhances the level of user's immersion. Another approach on designing immersive interactive 360-degree video experiences has been introduced through the integration of 360-degree video with hypervideo where links and annotations are integrated in the video in different times and areas of the 360-degree view [2]. In this case, innovative navigation mechanisms have been designed and proposed. Those mechanisms, as depicted in the figure below (figure 3), are:

- a drag interface to assist panning;

- the view area indicator in the form of a pie chart;

- a mini map with the planar projection of the video, and;

- hotspots indicators and thumbnails highlighting the current scene. 


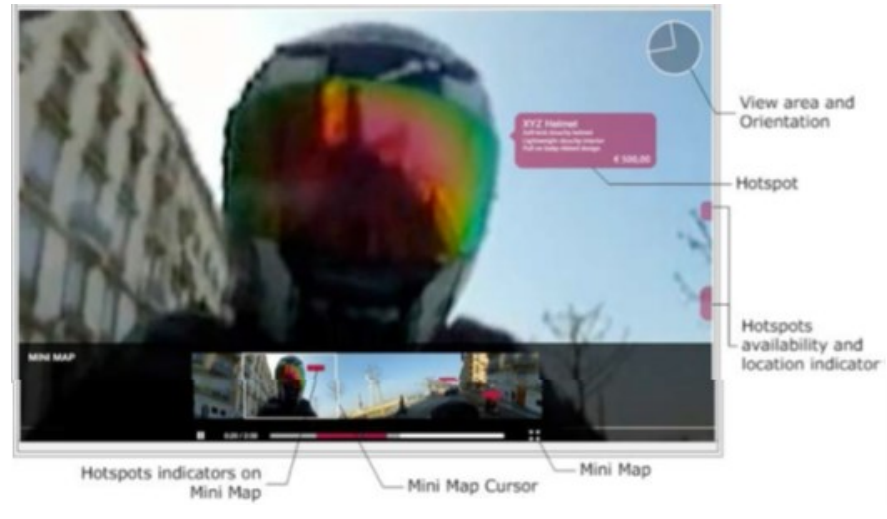

Fig. 3. Chambel, et al., HV $360^{\circ}$ Player in Mini Map Mode.

While this approach addresses some issues of enhancing interactivity and supporting navigation and orientation in 360degree video viewing on the web, the interface presented is complex and contains lots of information that may distract the user from the main story ending to a not such engaging experience. Finally, Chambel, et al. [1] point that research has to be done on enhancing entertainment and user engagement through storytelling mechanisms.

Cinematic VR has also moved effectively towards the production of storytelling 360-degree films introducing a new way of offering immersive experiences. Within [30] is a company that has released a series of short 360-degree films that have been seen in more than 40 countries and have been translated in 15 languages. This is indefinitely a successful example of how communicating stories can raise awareness as the user feels like he's actually taking part in the story. TV broadcasters, news channels and journalists are also looking of new forms of immersive media and live events broadcasting in VR as they allow the users to feel that they participate as spectators in a broadcasted event making the overall experience more immersive and engaging [13].

The question though still exists on how these experiences could be designed to be coupled with other devices (mobiles, head-mounted displays) making the experience more interactive, engaging and collaborative-social.

\section{CHALLENGES AND IMPLICATIONS}

There are several challenges and implications that have to be addressed when trying to create immersive, interactive and engaging experiences with the use of 360-degree videos. In this section we discuss two kinds of challenges, those that deal with technical implementations and those that refer to the overall design process. These are based on the limitations inflicted by the video itself which is used as the main game scene and lacks depth of virtual space where the user can navigate. In conventional virtual environments the user can navigate in complex 3D geometries reconstructing real spaces, attempting to simulate reality, or creating imaginative spaces that cannot exist in real life. Moreover, another sensitive issue that makes the design of interactions more complicated in 360-degree video applications is the sense of presence that the user should experience. The user's sense of presence can be enhanced by creating a natural and close to real environment and by avoiding incongruous visual cues allowing in this way smooth interaction in the virtual space. Our approach in addressing those challenges is to focus on designing strong, realistic, rich and cognitively demanding branching narratives with the use of 360-degree video. A list of technical and design challenges is presented below, that withdraw from our study and aims, followed by a proposed way to address them through the description of a design framework.

\section{A. Technical challenges}

\section{Smooth transition between video resources}

By integrating the branching narrative game mechanism, in order to design immersive interactive applications that are not disruptive, we have to face the challenge of possible delays and blinking effects when changing between the video resources that are used as the subplots of the game storyline. The transition between the video resources should be smooth and seamless in order to ensure an unbroken and consistent presentation of the game story. The users should be able to focus only on the story and the challenges presented and feel like they are being part of it forgetting that they play a game. Therefore any kind of meaningless interruption and distraction that can break the feeling of immersion must be avoided.

\section{Natural, close to real environment}

Besides the capturing and use of 360-degree video that is a real representation of the world, 3D objects can be also included in the environment in order to offer the feeling of interacting with the content. As the background scene is created through the projection of a video source, the appearance of the $3 \mathrm{D}$ objects incorporated in it should be as realistic as possible presenting a consistent and natural environment. For this reason the reconstruction and rendering of the objects should be first of all relevant to the video captured scene while representing a real model of the world.

\section{Reality-based navigation}

Navigation in video applications is mainly time-based using the forward or backward options to move between the frames or based on the camera movement support for pan, tilt and zoom [21]. The goal of new interaction designers should be to allow the user to perform realistic tasks naturally, to provide additional non real-world functionality, and to use analogies for these commands whenever possible [14]. In order for the users to feel that they are actually in the story, they actively participate, navigate and interact in it, the scene should mimic the real world.

Besides those three key technical challenges presented above that we are trying to address through our design approach that follows, there are also further implications that are worth to mention. Those implications have to be faced when designing collaborative gamified experiences. Collaborative gamified experiences have proven to offer higher levels of user engagement through social interaction. By offering social interaction to our users through multi-player gaming or video game live streaming we have to address the challenges for 360-degree video streaming on demand and therefore encoding implications. The 360-degree video file sizes are very large and they can be an impediment to delivering 360 video or VR in a quality manner at scale. There 
are three approaches on mapping 360-degree video on 3D objects for rendering; the spherical approach, the cubical approach and the pyramid approach as shown in figure 4 . The most popular one is that of the spherical approach for standard equirectangular layout which flattens the sphere around the viewer onto a 2D surface. But this approach causes several implications for streaming content without waiting for buffering and wrapping effects. The cubical approach is a combination of six faces of the cube, each face representing one direction relative to the viewer (up, down, left, right, front, and back). The cube can be used to map an entire $360^{\circ}$ degree video. Each frame of the $360^{\circ}$ video splits into six areas, each representing a viewing direction, apart from the down area, that that is replaced by the CGI terrain. Some benefits of the cubical approach are as follows: each face of the cube is treated equally and hence during rendering the quality of the video is not affected, so regardless of the where the player is looking at and the speed of changing their perspective the quality of the video remains the same; it reduces the size of video files by $25 \%$ compared to spherical projections. A pyramid approach works by mapping an equirectangular video onto a pyramid. In this approach only the base of the pyramid (what the viewer sees) is rendered in full resolution while the rest of the video gradually decreases in quality and wraps around the viewer. This approach results to reducing the file size of the videos by $80 \%$ compared to the spherical approach [6].

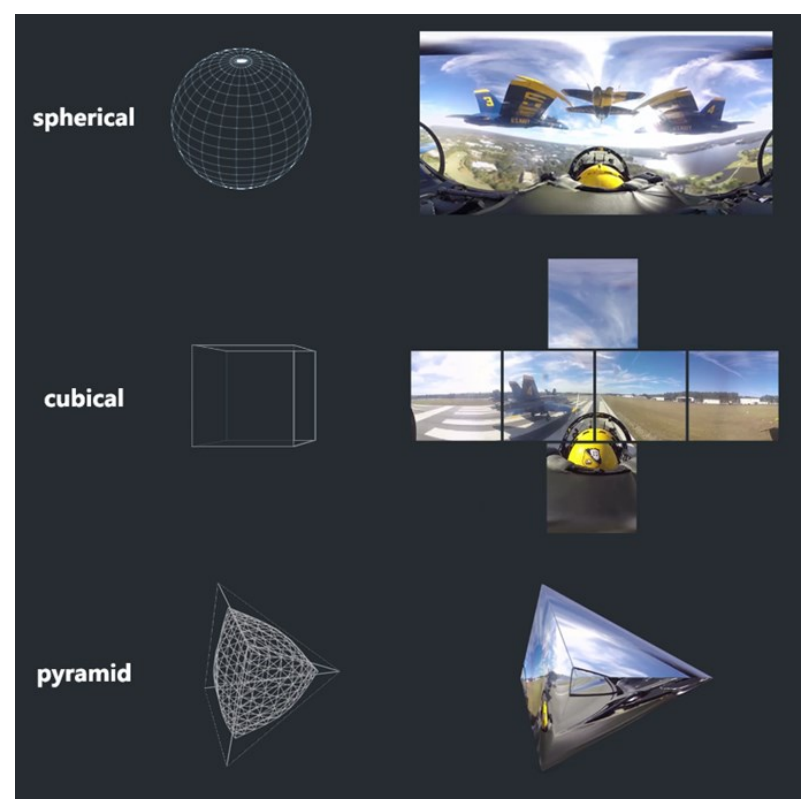

Fig. 4. 360-degree video mapping approaches.

\section{B. Design challenges}

1. Non-intrusive, non-distractive user interface design

The design of the graphical user interface in such experiences should also be as transparent and non-intrusive as possible giving the sense of an augmented view of the real scene with the minimum info required for running and controlling such experiences. Moreover, the interaction with the UI such as the use of menu and the display of any informative text should not pause the game scene and make it vanish from the player's view as this comes in contrast with our aim of offering immersive experiences.

\section{Navigation and orientation mechanisms}

Another important design challenge is the support of navigation and orientation in 360-degree video, as in panoramic view interested parts of the video could be out of the user's sight. As the users can freely navigate in the panoramic view, they may easily feel anxious they are missing something important [31]. In such rich environments consisted by 360 degree video, new navigation mechanisms should be designed and integrated to help user orientation in panoramic view while reducing the cognitive load.

\section{Gamified design}

Adopting techniques from the games design area can lead to more engaging and motivating experiences. There are various game mechanics and gamification techniques that can be integrated to the design of interactive applications in order to offer more fun and engaging experiences [8]. The challenge that has to be addressed in our case is to invent an effective combination of the right game mechanics that can be adopted to the design of 360-degree interactive video applications without diminishing the user's feeling of immersion.

\section{THE 360-DEGREE GAMIFICATION FRAMEWORK}

In order to address the technical and design challenges for more immersive and engaging experiences offered by consuming 360-degree video, a conceptual gamification framework for mixed reality applications is proposed. The framework is based on the use of game elements in a 360degree video environment to enhance user interaction with the medium. The presented framework, as depicted in the figure below (figure 5), is the basis for creating 360-degree gamified applications.

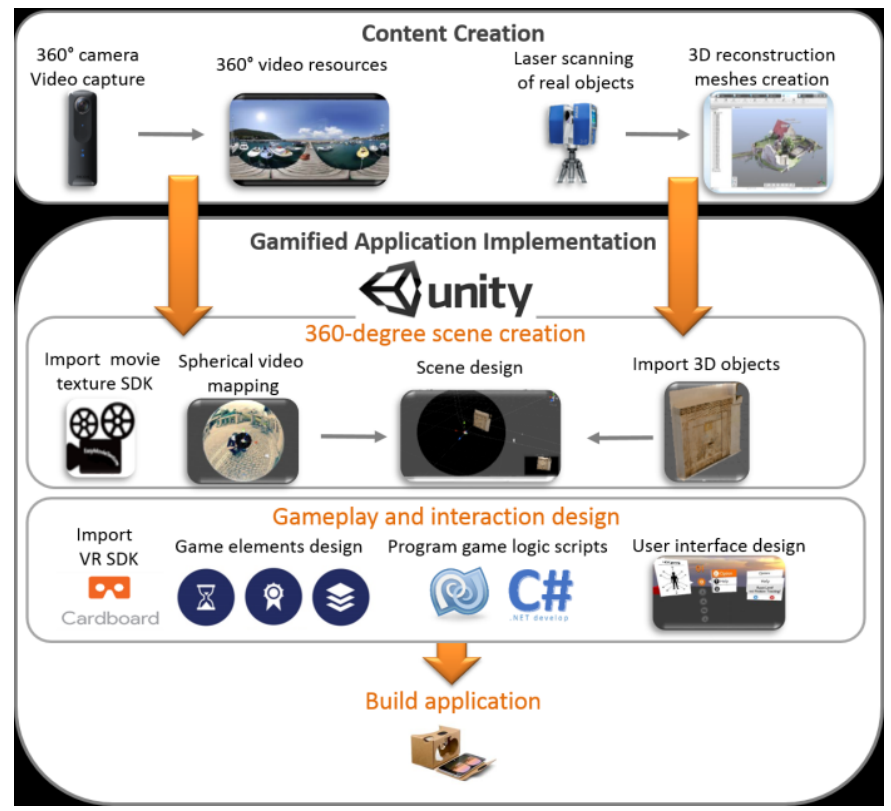

Fig. 5. 360-degree gamification framework.

The main core of the design framework is a game engine and in our case that of Unity 3D [28]. The first step is the 
creation of the content. 360-degree video resources must be produced using relative production cameras such as the lowcost and easy to use Ricoh Theta S [23], the Kodak PIXPRO SP360 Action Camera (shoots at $235^{\circ}$ actually, so you need two cameras to capture a full $360^{\circ}$ view) [15] or the more expensive, but with higher analysis, OMNI spherical video capturing solution offered by GoPro [11]. After the capturing process, the video resources are edited using the software tools offered by the camera production companies accordingly and categorized and annotated in order to create the branching narrative of the gaming experience. Moreover, the 3D objects that are necessary for the game design are also produced using laser scanning equipment (or camera equipment for photogrammetric reconstruction) to create the point clouds of real objects and a software tool to convert them to meshes.

The next step for designing 360-degree gamified immersive applications is the creation of the scene. For creating the 360degree game scene we follow the spherical approach to define a panoramic environmental surround. A game environment using a spherical approach is created by projecting an equirectangular video onto the surface of a 3D sphere that is inserted in the Unity project's scene and by placing the camera at the center of the sphere. 360-degree video resources are applied as movie texture on the sphere through the integration of a specific movie texture SDK (the Easy movie texture Unity asset). The specific asset allows the application of 360-degree videos as textures on a $3 \mathrm{D}$ object of the scene for targeting the build of mobile VR applications for Android or iOS. The scene design process continues by importing the $3 \mathrm{D}$ reconstructed game objects and placing them inside the sphere.

To start the implementation of our immersive application, the required SDK for building VR mobile applications must be integrated. In our case we target the use of low cost Google cardboard viewer devices, therefore the Google cardboard VR SDK [10] is loaded in the Unity $3 \mathrm{D}$ game engine. Then we move to the design and integration of our preferred game elements, such as time pressure mechanisms, badges assignment techniques and level-systems and the programming of the required game logic scripts to run the game scenarios. The user interface of the gamified application is also designed to support the user interaction with the content and the overall game logic, such as the display of notifications, the level/ points/badges visualizations and the navigation support view.

Below we present the way of addressing the technical and design challenges that have been outlined above (see section III).

\section{A. Addressing technical challenges}

Moving now to the design of the overall branching narrative experience we need to address the first challenge mentioned before, that of smooth transition between the video resources (see Section III.A). To design our 360-degree video narratives scene environment in Unity we can use screen fade-in and fade-out techniques between the disabling and enabling of the video resources to avoid the blinking effects. In order to avoid delays when changing on the fly from one video resource to another we can use different spherical objects in the scene where each video resource is already associated as a movie texture asset and use disable and enable techniques when moving from one to another. The videos should have been already preloaded and be in pause state in order to avoid delays from dynamically loading videos in the device's memory at the time they should be played.

Additionally, for enabling interaction with the scene and the content of our gamified applications, we use 3D objects that allow the user to interact with them and change their status and the status of the game. A series of 3D objects are placed in the internal space of each 3D sphere where the relative video resource of our narrative is associated. The $3 \mathrm{D}$ objects become visible to the user with the use of game's scene lights that are placed according to the lighting source of the real scene that has been video captured (sunlight source, building light sources etc.). In this way, we target a realistic lighting and shading of the $3 \mathrm{D}$ objects in the scene. Those game objects, their animation effects and actions triggered when interacting with them are essential for progressing in the game and addressing challenges to the players.

We therefore move to the creation of a mixed reality game environment combining video content and computer generated $3 \mathrm{D}$ elements. As a result, we have to address the second challenge described above of making the environment more natural and close to real (see Section III.A). For realistic reconstruction of objects we suggest the use of photogrammetry techniques for realistic reconstruction [12] or laser scanning for the creation of the objects point clouds which can then be converted to equivalent meshes. Photogrammetric reconstruction is a low cost method as it is based on the use of a large set of photos of the object but it is time-consuming as it needs a lot of time for the image processing algorithm to run. Laser scanning on the opposite is faster and more accurate, but the equipment required to scan and produce the objects' point clouds, which is the cluster of points with three dimensional information that will be converted to meshes, is 50 times more expensive. Moreover, the position and transformation of the objects in the scene should be adequate to the captured video scene. In case for example that the video shows the user to move to a specific direction of the captured area, the scale of the 3D objects placed in the scene should fluctuate according to the user's view. This is achieved with the use of the scripting API of Unity and the transform function for each object.

In addition, scripts are used for defining the game logic and designing interaction processes and events. A non-intrusive, non-distractive UI is also necessary to present the user progress in the game without braking immersion. The UI therefore is limited to display only the necessary information at each step of the game such as the level, the badges, the open challenges and the time remaining for completing those. UI elements should be also transparent and not placed in a camera point view that would distract the player from experiencing the key scenes of the video play.

Finally, in order to increase the user's feeling of presence, we need to address to third challenge of designing realitybased user navigation in the environment (see Section III.A). In the presented case of mixed media immersive applications we design an effect of virtual navigation in the 
video scene through the capturing and use of video resources in first person 360-degree view (at the average height of the targeted user) where the person is moving in the real world. Navigation in the game is achieved by playing a new video resource where the user, according to their choices, is shown to walk towards another area in the real world. In this way, the user has the sense of moving in the virtual scene through predefined paths that have been video captured.

\section{B. Addressing design challenges}

In order to address the need of designing a UI that is nonintrusive and non-distractive (see Section III.B) we have limited the controls to a simple Graphical User Interface (GUI) design, depicting only the necessary information required for the player to navigate and interact with the content in the scene. The GUI consists therefore of five key semi-transparent elements shown at the bottom and top corners of the screen: a mini map displaying the user's field-of-view and the 3D objects position in the 360-degree environment; a pop-up element displaying a question and three possible answers that the user can select and which appears when the a 3D object is selected; a graphical display of the time left to address a challenge; a graphical element displaying the user's score and level; and a video player pause/play button. Those elements are placed on the sides of the video screen and do not hide the main viewport or pause the action while they are used mainly to display information without making the VR environment disappear. The mini map GUI element addresses the second design challenge for integrating a suitable for 360-degree video applications navigation and orientation mechanism (see Section III.B). The users are presented with a 2D "radar" mini map element that continuously displays their viewport as also the relative position of the $3 \mathrm{D}$ objects of interest in the scene in order to assist them to navigate towards them.

Working towards an effective gamified design, a list of game mechanics are used starting with the most important one for our case which is the branching narrative (see Section II). Several 360-degree videos are captured in order provide enough content to allow the story to unfold according to the user selections. Challenges are continuously presented to the players at each level of the game that increase their interest while testing their knowledge and allowing them to apply it. Addressing challenges makes people feel they have earned their achievement giving them the sense of accomplishment which is one of the eight core drives of gamification according to the Octalysis gamification framework [3]. At each game level the user must address a specific challenge in order to gain points and advance in the game. Levels and goals help to map and show the users' progression in the game. The logic of the overall gameful experience is also designed based on the concept of exploratory games that allows the user to freely navigate and revisit several stages/levels of the game by experiencing different narratives till he identifies and completes all challenges presented [16]. The exploratory approach triggers the users' curiosity motivating them to master the rules and affordances of the game by supporting them to level-up and advance in the game, making the whole experience more engaging. Time pressure is another game element used through the time limit that corresponds to the length of the loaded 360-degree video resources. The users are presented with a specific challenge they have to complete before the end of the current video play. Limiting the amount of time people have to complete specific tasks can impact users focus on addressing the challenges presented. This concept of the specific time or period in which learners intensify their cognitive skills in order to achieve their learning goals has been referred to as reclaiming the learning time and creating the necessary conditions for the achieving the "learning momentum" [1]. It can also lead to different selections combined effectively with the branching narrative mechanism.

\section{CASE STUDY}

Our case study is based on the use of the 360-degree gamification framework presented earlier (see Section IV) to create an immersive educational application of a treasure hunt game in a cultural heritage site, that of the historical center of Rethymno city, in Greece. Rethymno city has been chosen because of its great historical importance, preserving artefacts and monuments in good condition from different historical periods such us the Ottoman and Venetian periods, useful for designing interesting branching narratives. The game has been developed to be used with the Google Cardboard VR device and an Android mobile phone. In order to create the gameplay, several video resources have been produced capturing the user's movement in 360-degrees view between the several key areas, historical buildings and artefacts on the site to be used for user navigation in the virtual scene. Moreover, a list of short-time 360-degree videos per area of interest where also captured in first person view depicting the user standing in the middle of each area or in front of a building, to allow the exploration of the place in 360-degree view. Those exploratory video resources are used to create a mixed media scene where 3D objects depicting important historical artefacts are integrated in order for the user to be able to identify them in the scene and move to another level of the game. The 3D objects that are used have been realistically reconstructed with the use of laser scanning equipment or photogrammetric techniques. Those $3 \mathrm{D}$ reconstructed objects represent important historical remains of the Ottoman period that can be found in the old city of Rethymno [4].

The game consists of a series of events that are shown in figure 6 which demonstrates the overall gameplay logic, the required user input and the GUI updates. The game starts by displaying the first 360-degree video short clip where the player stands at the middle of a square in the historical center of the city. The player has the role of an Ottoman soldier missioned to collect water from several historical fountains of the Ottoman period, which remain in the city, and carry it to the most important fountain of the city, the Rimondi fountain [24], in a specific time limit.

The player is presented through the UI with the first challenge asking to spot three historical fountains that are placed in the main square scene. The real position of those fountains is not actually in the main square but in other areas in the city. The scope of the game is to spot these fountains in the scene in order to later on place them at the Rimondi fountain. 


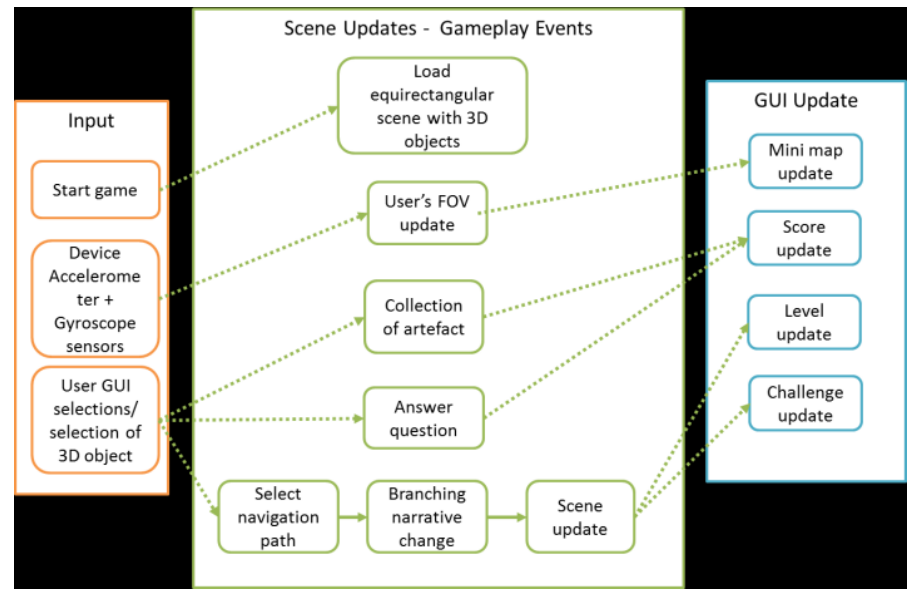

Fig. 6. Gameplay logic.

For the navigation in the scene the player is assisted through the 2D "radar" GUI map element showing the position of the fountains. The player spots a fountain by focusing on it for 5 seconds, till a marker appears indicating to select it using the Cardboard's button at the top right of the device. For each fountain the player gains 10 points and his score in the game is increased (water level indicator depicted though the UI). The user has to spot all fountains in the time limit specified by the video clip's duration and depicted with a chronometer game element running backwards (timer) to gain the extra points for accomplishing the challenge. If the player fails to accomplish the first challenge on time, the video clip loops again allowing the player to replay the session in order to progress to the next level without gaining any extra points in this level.

After collecting all the three fountains, the player is requested to peak one and select to move towards its real location through the shortest path between two possible options (branches). Thus two possible branching narratives are provided for each selected fountain. Depending on the selected path, players are presented with different historical information and/or clues contributed to the gameplay. The selected video clip is depicting the player walking in the chosen direction towards the area in the city where the selected fountain is actually placed. At the end of that video clip the player is in front of the selected fountain. Then the next video clip is played with a time limit to address the next challenge which is a historical question about the fountain. Through the UI the player is presented with the question and three possible answers. The question can be answered through either general knowledge or using information gained during his tour in the selected path.

If the players' first selection is correct they gain 20 points, if the second selection is correct they gain 10 points, otherwise the gain no points. At the end of this level, the water indicator increases demonstrating the points collected respectively. As a next step, the player must select another fountain element and a path from the presented ones to move to its real location. The procedure of gaining points and progressing again is the same as at the previous level. After having visited all the locations of the fountains in the city, answered the relative questions and collected an amount of water, the players are transferred to the location of the Rimondi fountain. This stage running at the area of the Rimondi fountain is the final stage of the game. At the final stage, the collected fountains can be placed on the three tabs of the Rimondi after answering a historical question again for each one of them. When a correct answer is given, the related fountain is depicted as checked in the screen and the relative tab area is shown as filled with water. When all questions are answered and all tabs are filled with water, the game ends and the players are addressed with a Gold, a Silver or a Bronze badge of the Ottoman citizen of Rethymno according to the points collected.

The players can always replay the game starting from the first level again trying to collect the maximum number of points that can be assigned to each level and exploring different tours in the city by selecting other paths.

A snapshot of the gameplay is shown at figure 7 depicting the main UI elements, the radar map, the water level indicator, the time limit and the play/pause button. The player is at the first stage of the game which takes place at the main square having selected one $3 \mathrm{D}$ fountain object of the spotted ones. The score is depicted also though the water level indicator. The next step is to choose a path, selecting to move forward or on the left side to visit the real location of the fountain.

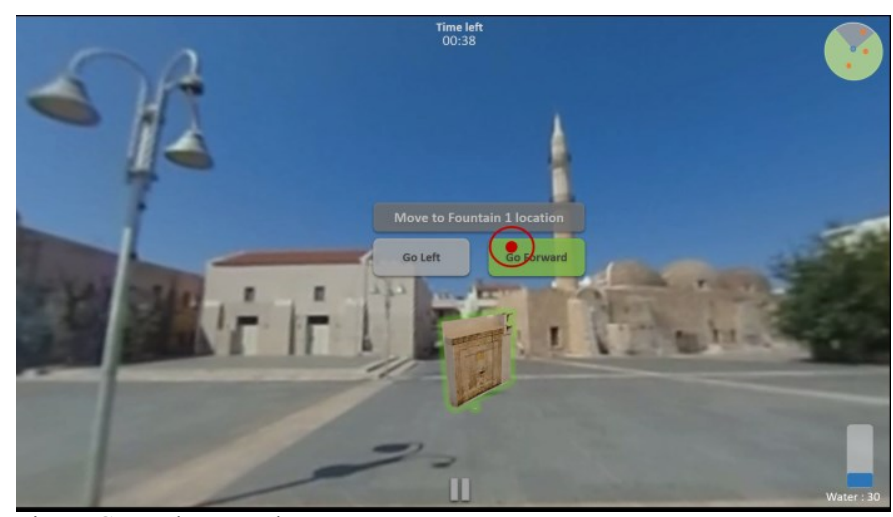

Fig. 7: Gameplay snapshot.

\section{DISCUSSION AND FUTURE DIRECTIONS}

In order to design engaging experiences and increase the level of user's immersion within virtual worlds built with the use of 360-degree video, specific guidelines that can address the presented challenges should be investigated and defined. The presented gamification framework is a first approach towards this direction.

To offer the user the expected feeling of immersion and sense of actually participating in the game story, an interesting story game that unfolds through 360-degree videos has been designed. The main game story has been based on the branching narrative technique presenting a virtual scenario where the user has the option though interacting with the environment to experience it in different ways. This technique offers the user an enhanced sense of presence as it creates the feeling of controlling and actually taking part in the scenario. Good stories attract the user to the gaming experience while make the world seem more believable. A list of other game mechanics have been integrated in the design of the gaming experience and the UI, such as revealing challenges accompanied by time pressure techniques and the assignment 
of points, levels and badges that engage the user by giving the sense of accomplishment and progression in the game. Navigation and orientation mechanisms have been also used through the incorporation in the UI design of 360-degree "radar" maps depicting the field of user's view in real time and showing the position of points of interest in the scene. Techniques for smooth transition between the video resources have been followed and the gaming environment has been designed to be as close to real as possible depicting realistic interaction.

The graphical user interface has been carefully designed to be transparent and non-intrusive giving the sense of an augmented view of the real scene.

All the above presented and incorporated design techniques to our case study should be evaluated in the future through usability studies involving real users. Real user studies will provide us with the necessary results in evaluating our approach for addressing the identified list of challenges.

\section{ACKNOWLEDGMENT}

The research that led to this paper was financially supported by the University of Westminster through a $\mathrm{PhD}$ studentship.

\section{REFERENCES}

[1] Bouki, V. and Economou, D. Using Serious Games in Higher Education: Reclaiming the Learning Time Immersive Learning Research Network Conference Prague, Czech Republic 13 Jul 2015 IOS Press

[2] Chambel, T., Chhaganlal, M. N., \& Neng, L. A. Towards immersive interactive video through 360 hypervideo. In Proceedings of the 8th International Conference on Advances in Computer Entertainment Technology (p. 78). ACM, 2011.

[3] Chou, Y. Octalysis: Complete Gamification Framework. Yu-Kai Chou \& Gamification blog, available at: http://goo.gl/Qa1 Vm2, last accessed on $23 / 06 / 2016$.

[4] Digital Crete online database by IMS FORTH, Ottoman monuments directory listing, available at http://digitalcrete.ims.forth.gr/tourkology_monuments_search.php?1=1, last accessed on 03/07/2016.

[5] Douglas, J. Yellowlees \& Hargadon, Andrew. (2004) The Pleasure of Immersion and Interaction: Schemas, Scripts, and the Fifth Business. In: Wardrip-Fruin, Noah \& Harrigan, Pat (Eds.) First Person: New Media as story, Performance, and Game. Cambridge, MIT Press.

[6] Facebook Engineering post : Next generation video encoding techniques for 360 video and VR, available at: https://code.facebook.com/posts/1126354007399553/next-generationvideo-encoding-techniques-for-360-video-and-vr/, last accessed on $15 / 06 / 2016$

[7] Fortt McMoney 360-degree interactive storytelling, available at http://fortmcmoney.com/en/\#/fortmcmoney, last accessed on 04/06/2016.

[8] Gamified UK, Gamification elements, mechanics and ideas, available at: http://www.gamified.uk/2015/02/04/47-gamification-elementsmechanics-and-ideas/, last accessed on 29/06/2016.

[9] Gaver, W. W., Bowers, J., Boucher, A., et al. The drift table: designing for ludic engagement. Proc. CHI EA '04 ACM Press (2004), 885-900.

[10] Google Cardboard VR SDK for Unity, available at: https://developers.google.com/vr/unity/, last accessed on 04/06/2016.

[11] GoPro VR OMNI camera systen for spherical video capturing, available at: https://vr.gopro.com/, last accessed on 05/07/2016.
[12] Grün, Armin, Fabio Remondino, and Li Zhang. "Photogrammetric reconstruction of the great Buddha of Bamiyan, Afghanistan." The Photogrammetric Record 19.107 (2004): 177-199.

[13] Hayden S., Belgian news channel hints future immersive newscasts, available at: http://www.roadtovr.com/belgian-news-channel-hintsfuture-immersive-newscasts/, posted on 15/02/2016.

[14] Jacob, R.J.K., Girouard, A., Hirshfield, L.M., Horn, M.S., Shaer, O., Solovey, E.T. and Zigelbaum, J., Reality-Based Interaction: Unifying the New Generation of Interaction Styles (Work in Progress paper). in CHI 2007, (2007), 2465-2470.

[15] Kodak pixpro SP360 Action camera for $360^{\circ}$ video capture, available at: http://kodakpixpro.com/Americas/cameras/actioncam/sp360/, last accessed on 10/06/2016.

[16] Linda de Valk, Pepijn Rijnbout, Tilde Bekker, Berry Eggen, Mark de Graaf, and Ben Schouten. Designing for playful experiences in openended intelligent play environments. In IADIS International Conference Games and Entertainment Technologies, pages 3-10, 2012.

[17] Madigan Jamie, The Psychology of Video Game Immersion, available at: https://www.psychologytoday.com/blog/mind-games/201207/thepsychology-video-game-immersion, posted on Jul 03, 2012.

[18] Marczewski, Andrzej. Gamification: a simple introduction. Andrzej Marczewski, 2013.

[19] O'Brien: Get Ready for the Decade of Gamification. San Jose Mercury News. October 24, 2010, available at: http://goo.gl/4XQp2c, last accessed on 19/04/2016.

[20] Oculus Rift head-mounted display device - Next-generation virtual reality, available at: https://goo.gl/ZVwzEb, last accessed on 22/05/2016.

[21] Petry B., and Huber J., Towards effective interaction with omnidirectional videos using immersive virtual reality headsets. In Proceedings of the 6th Augmented Human International Conference (AH'15). ACM, New York, NY, 217-218, 2015.

[22] Reeves, B. and Read, J.L. Total Engagement: Using Games and Virtual Worlds to Change the Way People Work and Businesses Compete. Harvard Business School Press, Boston, MA, 2009.

[23] Ricoh Theta S $360^{\circ}$ video camera, available at: https://theta360.com/uk/about/theta/s.html, last accessed on 08/06/2016.

[24] Rimondi fountain Cultural Heritage Monument, Municipality of Rethymno, information available at: https://www.rethymno.gr/en/city/Rimondi/rimondi.html, last accessed on 27/06/2016.

[25] Rollings, Andrew; Ernest Adams. Fundamentals of Game Design. Prentice Hall. pp. 194-204, 2006

[26] Slater, M. \& Usoh, M., Presence in Immersive Virtual Environments. Proceedings of the IEEE Conference - Virtual Reality Annual International Symposium (VRAIS'93), IEEE Neural Networks Council, Seattle, WA, USA., September 18-22, IEEE Computer Society, pp. 9096, 1993.

[27] Smolic, Aljoscha, Karsten Mueller, Philipp Merkle, Christoph Fehn, Peter Kauff, Peter Eisert, and Thomas Wiegand. "3D video and free viewpoint video-technologies, applications and MPEG standards." In 2006 IEEE International Conference on Multimedia and Expo, pp. 21612164. IEEE, 2006.

[28] Unity 3D Game Platform, available at: http://unity3d.com, last accessed on $27 / 05 / 2016$.

[29] Wirth, W., hartmann, T., Bocking, S., Vorderer, P., Klimmt, C., Holger, S., Saari, T., Laarni, J., Ravaja, N., Gouveia, F., Biocca, F., Sacau, A. Jancke, L., Baumgartner, T., \& Jancke, P. (2007). A Process Model for the Formation of Spatial Presence Experiences. Media Psychology, 9, 493-525.C. Goodwin, "Gestures as a Resource for the Organisation of Mutual Orientation", in Semiotica, vol. 62(1/2), 1986, pp. $29-49$.

[30] Within, Storytelling VR films production company, available at: http://with.in/\#films, last accesed on 20/06/2016.

[31] Zoric, G., Barkhuus, L., Engström, A., and Önnevall, E. Panoramic Video: Design Challenges and Implications for Content Interaction. Proceedings of the 11th European Conference on Interactive TV and Video, ACM (2013), 153-162.P. Ekman and W. Fiesen, "Facial Action Coding System", Palo Alto, CA: Consulting Psychologists Press, 1978. 